\title{
Scanning Ion Conductance Microscopy Reveals Differences in the lonic Environments of Gram-Positive and Negative Bacteria
}

\author{
Kelsey Cremin, Bryn A. Jones, James Teahan, Gabriel N. Meloni, David Perry, Christian Zerfass, \\ Munehiro Asally, Orkun S. Soyer,* and Patrick R. Unwin*
}

Cite This: Anal. Chem. 2020, 92, 16024-16032

Read Online

\section{ACCESS |}

山lll Metrics \& More

Article Recommendations

Supporting Information

ABSTRACT: This paper reports on the use of scanning ion conductance microscopy (SICM) to locally map the ionic properties and charge environment of two live bacterial strains: the Gram-negative Escherichia coli and the Gram-positive Bacillus subtilis. SICM results find heterogeneities across the bacterial surface and significant differences among the Gram-positive and Gram-negative bacteria. The bioelectrical environment of the $B$. subtilis was found to be considerably more negatively charged compared to E. coli. SICM measurements, fitted to a simplified finite element method (FEM) model, revealed surface charge values of -80 to $-140 \mathrm{mC} \mathrm{m}^{-2}$ for the Gram-negative E. coli. The Gram-positive B. subtilis show a much higher conductivity around

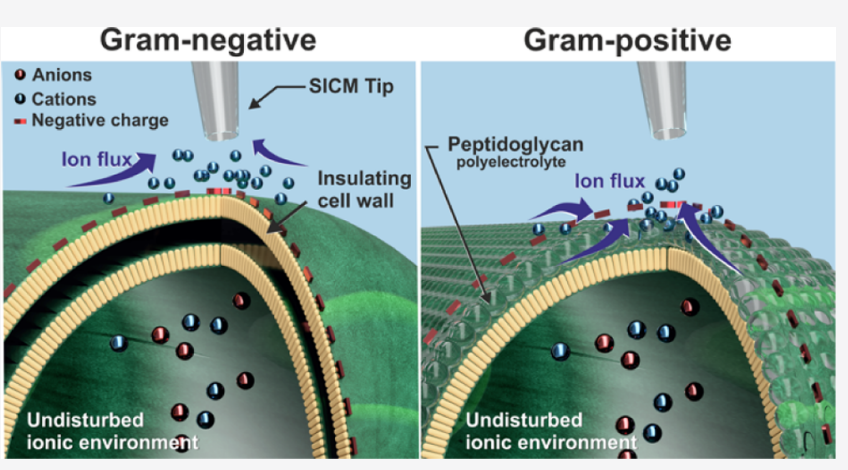
the cell wall, and surface charge values between -350 and -450 $\mathrm{mC} \mathrm{m} \mathrm{m}^{-2}$ were found using the same simplified model. SICM was also able to detect regions of high negative charge near B. subtilis, not detected in the topographical SICM response and attributed to the extracellular polymeric substance. To further explore how the B. subtilis cell wall structure can influence the SICM current response, a more comprehensive FEM model, accounting for the physical properties of the Gram-positive cell wall, was developed. The new model provides a more realistic description of the cell wall and allows investigation of the relation between its key properties and SICM currents, building foundations to further investigate and improve understanding of the Gram-positive cellular microenvironment.

\section{INTRODUCTION}

Scanning ion conductance microscopy (SICM) is proving to be increasingly versatile for nanoscale functional mapping of live biological entities in situ to provide an abundance of information about the surface topography, ${ }^{1,2}$ together with interfacial properties, ${ }^{3}$ cell junction permeability, ${ }^{4-7}$ and dynamic $_{\text {processes. }}{ }^{8-12}$ SICM utilizes a glass or quartz nanopipette, filled with electrolytic solution, to probe a substrate immersed in an electrolyte bath. A bias is applied between two quasi-reference counter electrodes (QRCEs); one in the nanopipette and the other in the bulk solution to drive an ionic current through the nanopipette. The current magnitude is highly sensitive to nanopipette-substrate separation and local ion conductivity, including that arising from the double layer at charged interfaces, and can be used to probe (independently) topography and surface charge of a range of substrates. ${ }^{13-15}$

Critical information about the ionic environment of the substrate-solution interface can be acquired by employing specific potential-time SICM scanning protocols, ${ }^{16,17}$ in tandem with finite element method (FEM) simulations, as exemplified by the quantitative analysis of surface charge $^{15,16,18-20}$ and for reaction mapping. ${ }^{12,21}$ In this paper, we consider the use of SICM to characterize the ionic (bioelectrical) environment of single live bacterial cells. There is increasing interest in characterizing the bacterial cell microenvironment, ${ }^{22}$ as membrane properties and extracellular charge distributions are considered to play a role in biofilm formation, ${ }^{23}$ nutrient uptake, ${ }^{24}$ and cell differentiation (e.g. persister cells and biofilm forming cells). ${ }^{25,26}$

The bacterial microenvironment is expected to be directly influenced by the bacterial cell wall through surface appendages and proteins, as well as cellular secretions. The cell wall of bacteria can be broadly classified into two polyphyletic groups; Gram-positive and Gram-negative (Figure 1 ), each arising from structural differences which underpin the bacterial interactions with the environment. ${ }^{27}$ As seen in Figure 1, Gram-negative cell walls have an additional membrane covered in lipopolysaccharides, while Gram-positive

Received: August 27, 2020

Accepted: November 16, 2020

Published: November 26, 2020 


\section{Gram-Positive}

Gram-Negative

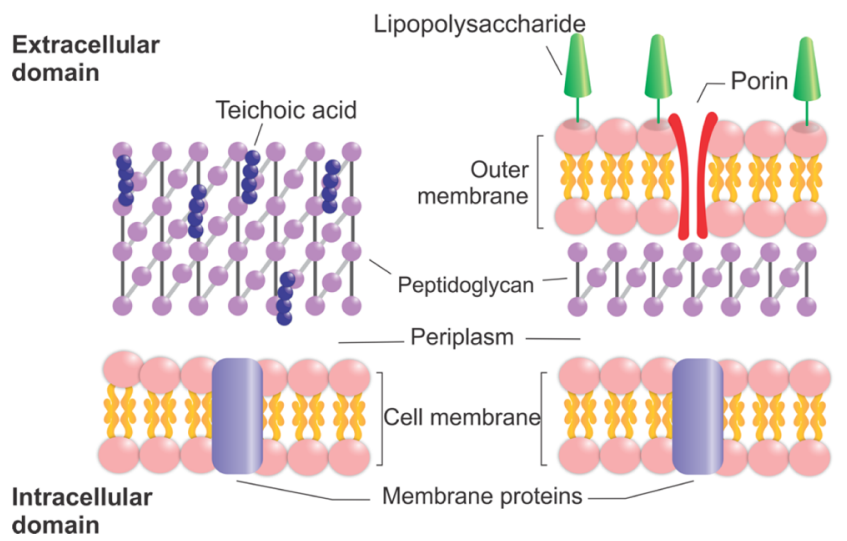

Figure 1. Cartoon illustration of bacterial cell envelope highlighting its key components in Gram-positive and Gram-negative bacteria.

cell walls contain only one membrane and have a thicker layer of peptidoglycan containing negatively charged teichoic acids. $^{28}$

Previous zeta-potential and electrophoretic measurements have shown that Gram-positive and Gram-negative bacteria both have a net negative surface charge, but the magnitude of the charge density is different between these two broad groups and between individual species within the groups. ${ }^{29-31}$ Zetapotential measurements determine the electric charge at the shear plane of the solid-liquid interface and do not provide a holistic descriptor of the charge environment at the cell wall, including important factors such as ionic permeability. Furthermore, the zeta-potential is measured for a population of cells and is blind to possible heterogeneities between different cells or across the cell surface of a single bacterium. Atomic force microscopy (AFM) can be applied to surface charge mapping of single cells, ${ }^{32}$ as carried out for microbial cells. ${ }^{33}$ AFM is only sensitive to the charge environment of the outermost surface, whereas with SICM, there is the possibility of probing ion permeability of a sample. ${ }^{4,34}$ Furthermore, for non-invasive scanning of soft live biological samples, the contactless nature of SICM can avoid deformation of the sample surface, while the lateral movement of the AFM cantilever can deform the live cells. In comparison to AFM, it has been demonstrated that SICM scans can provide better spatial resolution, along with a longer viable experimental time scale. $^{35,36}$

Here, we use SICM to map the charge environment of two different bacterial species, the Gram-negative Escherichia coli and Gram-positive Bacillus subtilis. Concurrent to these measurements, we develop a model of the properties and mechanisms influencing the interfacial charge and ion fluxes at and around the bacterial cell wall and perform FEM simulations to analyze model behavior against the experimental data. Our overarching aims are to demonstrate the application of SICM to single cells of live bacteria, so as to better quantify the single cell microenvironment in Gram-positive and Gramnegative bacteria and to provide a foundation that will enable future analyzes that can link diverse cellular behaviors to ionic dynamics within the cell-microenvironment interface.

\section{EXPERIMENTAL METHODS}

Chemicals. All reagents were of analytical grade and used without further purification. Deionized water (Milli-Q resistivity ca. $18.2 \mathrm{M} \Omega \mathrm{cm}$ at $25{ }^{\circ} \mathrm{C}$ ) was used in the preparation of all solutions. $50 \mathrm{mM}$ potassium chloride, buffered at $\mathrm{pH} 7.0$ with tris(hydroxymethyl)aminomethane (both from Sigma-Aldrich), was used as a supporting electrolyte for all of the SICM experiments. Low melting point agarose (Cleaver Scientific, CSL-LMA100), poly-L-lysine (PLL) (Sigma-Aldrich), or Cell-Tak (Corning) was used to adhere bacteria to glass slides, with the use of each defined herein.

Bacterial Cultures. B. subtilis [NCIB 3610- 4 hag depleted (created by Daniel Kearns and obtained from Munehiro Asally, University of Warwick) $]^{37}$ and E. coli K12 [wild-type, obtained from DSMZ (DSM no. 498)] were cultured in a modified M9 media containing $0.4 \% \mathrm{w} / \mathrm{v}$ glucose-full strain, and media composition can be found in the Supporting Information (SI), Section SI-1 and 2. The B. subtilis strain is a mutant that cannot produce flagella and hence has limited mobility, helping in cell adhesion. Bacteria were taken from freezer stocks (50\% glycerol, $-80{ }^{\circ} \mathrm{C}$ ) and grown in $40 \mathrm{~mL}$ volumes of media in sterile Erlenmeyer flasks. The cultures were grown overnight prior to SICM measurements on a shaking incubator at $37{ }^{\circ} \mathrm{C}$ and $150 \mathrm{rpm}$.

Bacterial Substrates. It is crucial for successful SICM scanning that the bacteria are adhered to a surface and is nonmotile. Achieving bacterial adhesion is known to be tasking, and several protocols are reported. ${ }^{38}$ In this work, several different adhesion methods were found to be suitable for anchoring and restricting bacterial movement, while not inhibiting culture survival, as detailed in Sections SI-3 and SI-4. In brief, adhesive layers of PLL, Cell-Tak, or $<0.5 \mathrm{~mm}$ agarose gels were deposited on the glass surface of a $50 \mathrm{~mm}$ glass bottomed dish (WillCo Wells, USA, HBST-5040). A 100 $\mu \mathrm{L}$ of aliquot of an overnight culture (optical density at 600$0.45 \mathrm{~nm}$ ) was drop cast to the adhesive substrate of choice. The sample was then left for $30 \mathrm{~min}$ at room temperature to adhere, followed by one gentle $10 \mathrm{~mL}$ application of clean media, which was then withdrawn by the Pasteur pipette to remove any unadhered bacteria.

SICM Scanning Regimes. Nanopipettes utilized as the scanning probes for SICM were fabricated using standard protocols and characterized by electron microscopy (EM). Fabrication and characterization protocols are described in Section SI-5. The end lumen radius of the nanopipettes was typically in the range $85-100 \mathrm{~nm}$ (measured accurately by EM). For SICM mapping, described in detail elsewhere, ${ }^{15,16,18,19}$ the nanopipette current was recorded continuously, while the nanopipette position and/or potential varied synchronously. The nanopipette was approached toward the substrate/bacterial cell surface at a predefined position with a small applied bias between the internal and bulk QRCEs $\left(V_{\text {Tip }}\right.$, Figure $\left.2 \mathrm{~A}\right)$, typically $50 \mathrm{mV}$, where the ionic current response is primarily sensitive to nanopipette-substrate separation (Figure 2B, period I). ${ }^{39}$ Upon a $2 \%$ decrease in current magnitude compared to the bulk value, corresponding to a nanopipette-substrate separation of tens of nanometer, as determined from FEM simulations (further explained in Section SI-6), the nanopipette movement stopped automatically, with the corresponding $z$-position (at each $x-y$ position) revealing the substrate topography. With the nanopipette at this position, $V_{\text {Tip }}$ was either pulsed to $-500 \mathrm{mV}$ for a defined period; or scanned linearly to $-500 \mathrm{mV}$, reversed linearly to $500 \mathrm{mV}$, and then back to the approach potential of $50 \mathrm{mV}$ (Figure 2B, period II) before retracting the nanopipette by 4 

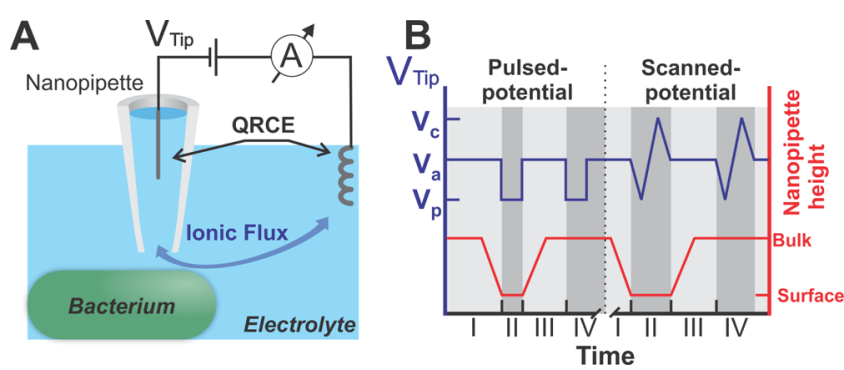

Figure 2. (A) SICM schematic depicting a nanopipette probe in the electrolyte and connected across two QRCEs, where $V_{\text {Tip }}$ is the potential applied to the nanopipette QRCE with respect to the QRCE in bulk solution. (B) Infographic to show nanopipette height in red (right $y$-axis), alongside $V_{\text {Tip }}$ (in blue, left $y$-axis) as a function of time, as applied at each position in an SICM map. Two different $V_{\text {Tip }}$-time profiles were applied, as shown on the left and right halves of the graph; pulsed- (left) and scanned-potential (right). The $V_{\text {Tip }}$ potentials defined are: $V_{a}$, the approach potential; $V_{\mathrm{p}}$, the pulsedpotential (and one limit of the triangular sweep); and $V_{\mathcal{c}}$ the other limit of the linear cyclic potential sweep. For most experiments, $V_{\mathrm{a}}=$ $50 \mathrm{mV}, V_{\mathrm{p}}=-500 \mathrm{mV}$, and $V_{\mathrm{c}}=500 \mathrm{mV}$ (for more information, see the text).

$\mu \mathrm{m}$, back into the bulk solution. The same potential pulsed or cyclic linear sweep program was repeated in the bulk (Figure 2B III and IV) to allow each surface electrochemical measurement to be normalized against a bulk measurement at each position (position-level self-referencing). ${ }^{16,40}$ Normalized current data are presented herein, where the near-surface current (at approx. $100 \mathrm{~nm}$ from substrate) values were divided by their corresponding bulk current (at approx. $4 \mu \mathrm{m}$ from substrate) values. For the pulsed-potential regime, this normalization was carried out using data from the end of the respective pulsed-potential period, while for the potential scans each datapoint in the current-potential plot at the surface was normalized by the equivalent scan datapoint obtained in bulk solution. The data presented herein are representative of 16 individual B. subtilis NCIB 3610- $\Delta$ hag depleted cells and 10 E. coli cells that were imaged during the course of these studies.

FEM Simulations. Two-dimensional (2D) axisymmetric models of the nanopipette in bulk solution and near the substrate were constructed in COMSOL Multiphysics (v. 5.4) with the Transport of Diluted Species, Laminar Flow, and Electrostatics modules. For converting experimental nanopipette currents to surface charge values, the real nanopipette geometry, acquired by EM and experimental conditions, were used in the FEM models. All other simulations exploring the influence of the substrate properties on the nanopipette current response were performed utilizing a representative (average) nanopipette geometry with a lumen radius of $95 \mathrm{~nm}$. Details and models are provided in Section SI-6.

\section{RESULTS AND DISCUSSION}

Probing the Gram-Negative Cell Envelope with Pulsed-Potential SICM. Pulsed-potential SICM was applied to map live E. coli cells adhered to PLL (Section SI-3) in a 50 $\mathrm{mM} \mathrm{KCl} \mathrm{electrolyte.} \mathrm{Typical} \mathrm{results} \mathrm{are} \mathrm{shown} \mathrm{in} \mathrm{Figure} \mathrm{3,}$ where (A) shows the topographical map of the bacterium, which is approximately $2 \mu \mathrm{m}$ in length and $1 \mu \mathrm{m}$ in height, in agreement with the literature values and the scanning EM images taken of cells at this growth stage (Section SI-7).$^{41}$ In the pulsed-potential stage, normalized nanopipette current values were in the range of $0.97-1.05$ (Figure 3B). The

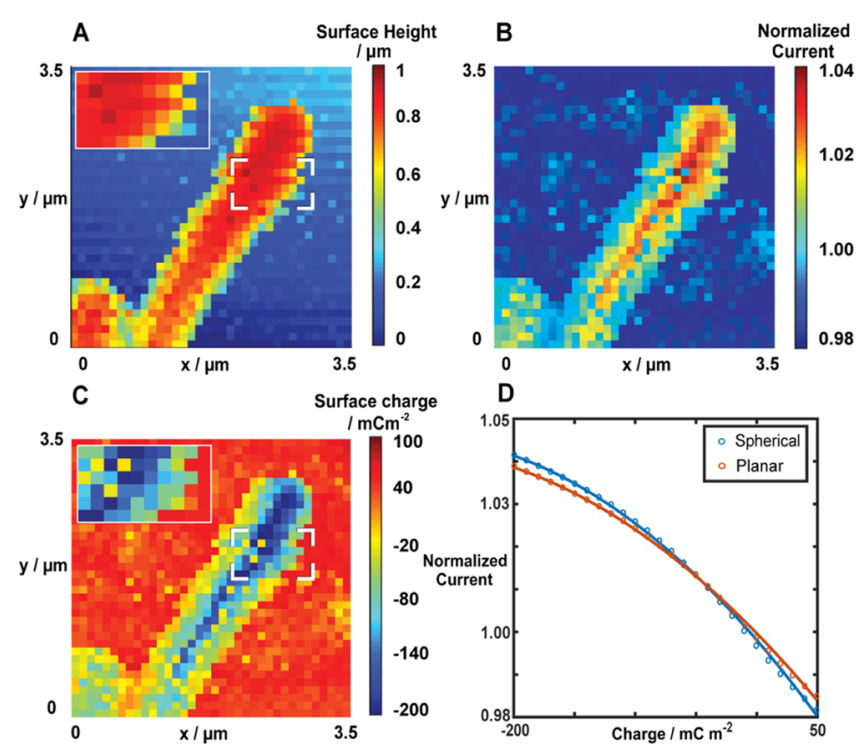

Figure 3. SICM topography (A), normalized current (B), and local charge density $\left(\mathrm{mC} \mathrm{m}^{-2}\right)$ following FEM simulation (C) maps of E. coli collected concurrently, where the inset expands the heterogeneous pixels discussed in the text. (D) Comparison of the FEM model simulation results using a planar (red) and spherical (blue) cell form (see Section SI-6). Lines are fitted calibrations through the simulated points. SICM experiment conditions: $50 \mathrm{mM}$ tris buffered $\mathrm{KCl}$ at $\mathrm{pH}$ 7, bacteria adhered to PLL. $V_{\mathrm{p}}=-500 \mathrm{mV}$ and $V_{\mathrm{a}}=50$ utilizing a $2 \%$ feedback current threshold (see the Experimental Methods section). Nanopipette inner radius of $93 \mathrm{~nm}$, with $100 \mathrm{~nm}$ hopping steps (pixel separation). Scan time of $80 \mathrm{~min}$. Images are raw data with no interpolation.

measured current values were converted to surface charge values using a FEM model (Section SI-6) that treated the bacterial wall surface as a planar charged impermeable insulator, reflecting the exterior membrane of Gram-negative bacteria (Figure 1). ${ }^{19}$ This model-based conversion resulted in a negative surface charge density of -80 to $-140 \mathrm{mC} \mathrm{m}^{-2}$ over the surface of the E. coli cells (Figure $3 \mathrm{C}$ ) and a positive surface charge of +30 to $+50 \mathrm{mC} \mathrm{m}^{-2}$ over the PLL. Interestingly, a significant degree of variation of the surface charge can be observed over the bacterium, where the central apex of the bacterium generates a higher normalized current (and therefore a more negative charge) than that at its edges (Figure 3B,C).

While the observed differences in current between the apex and edges of the bacteria are clear, as seen in Figure 3B, the corresponding surface charge differences could be affected by the model-based conversion of the experimental data. To explore this possibility, we modeled the charged surface as both a sphere (radius $0.5 \mu \mathrm{m}$, commensurate with that of the bacterium) and a planar surface, with the nanopipette set above in both cases (see Section SI-6 for more details of the models). In the corresponding FEM simulations using these two different models, the nanopipette-substrate distance was set for the same current set point (a $2 \%$ decrease in current from the bulk value), that is the same overall gap resistance. A comparison of the normalized current versus surface charge resulting from the two models is shown in Figure 3D. Overall, the difference between the models is small, with a maximum of ca. 0.002 difference in normalized current values at the most negative surface charge considered $\left(-200 \mathrm{mC} \mathrm{m}^{-2}\right)$. When calibrated to the experimental scan data over the bacterium in 
Figure 3 , the charge value calculated from the planar model was found to be at most $c a .10 \mathrm{mC} \mathrm{m}^{-2}$, more negative compared to the spherical model (see Section SI-8), which is negligible compared to the range of values $(-80$ to $-140 \mathrm{mC}$ $\mathrm{m}^{-2}$ ) observed over the cells. As the difference between the models is relatively small and the experimental scans include the flat background substrate, the planar model is used herein when describing the Gram-negative bacterial scans. Note that although COMSOL Multiphysics can, in principle, be used for three-dimensional simulations, ${ }^{21,42,43}$ the accuracy can be compromised and/or simulations become computationally expensive for complex problems, such as in this case, where there is an interplay of ion migration and electroosmotic fluid flow, and there is a need to consider charged interfaces. We thus retained a $2 \mathrm{D}$ model to assess the effect of a curved interface. The choice of a spherical object to assess the curvature of the substrate also maximizes the effect compared to the real situation where, for example, the majority of the bacterium is approximately cylindrical (Figure 3A). Further evidence that the significant differences in charge between the top and sides of the bacterium are not topographical in origin also comes from the fact that the generally much higher negative charge density $\left(\leq-100 \mathrm{mC} \mathrm{m}^{-2}\right)$ at the top of the bacterium extends right to the very end of the bacterium where there is significant curvature (Figure $3 \mathrm{~A}-\mathrm{C}$ ). ${ }^{44}$

Figure 3 also shows that there are small patches of low negative charge (with values of $c a .-20 \mathrm{mC} \mathrm{m}^{-2}$ ) within areas of much larger negative charge density $\left(\leq-100 \mathrm{mC} \mathrm{m}^{-2}\right)$; see for example the magnified insets in Figure 3C, where there are four such pixels. These are not "false approaches" of the nanopipette as the topography (height) difference between these pixels with the surrounding pixels is $c a .40 \mathrm{~nm}$ in two cases, and $<10 \mathrm{~nm}$ in the two other cases (inset in Figure 3A), similar to the topography variation from pixel to pixel in other regions of the cell. We considered whether some of these significant local variations in surface charge could be due to the SICM tip causing potential changes near the bacterial cell wall and activating voltage-gated or mechanosensitive ion channels (MSCs). To explore this possibility, we used the experimentally determined nanopipette geometry with FEM simulations to analyze potential field around the nanopipette tip (Figure 4). We find that the majority of the tip-induced voltage drop is in the first few microns within the nanopipette (Figure 4A), ${ }^{16}$ presumably due to the high resistance at the nanopipette lumen (140 M , calculated from $i-V$ curves recorded in bulk solution). Although some of the potential field extends from the end of the nanopipette, the impact of this on the bacterial surface, at the highest pulsed nanopipette potential of $-500 \mathrm{mV}$, is expected to be only -15 to $-25 \mathrm{mV}$ (Figure 4B), considerably less than the $-50 \mathrm{mV}$ depolarization potentials reported, for example, for E. coli $\mathrm{K}^{+}$ion channels. ${ }^{45}$ Unlike voltage-gated ion channels, MSCs are sensitive to both potential and mechanical stresses which can arise from external pressure. ${ }^{46}$ We thus further analyzed the FEM simulations to account for the pressure exerted by electroosmotic fluid flow from the nanopipette to the cell surface (discussed in Section SI-8). We found this to be $c a$. $-45 \mathrm{~Pa}$, suggesting a less than $10 \%$ probability of opening MSCs at the interfacial potential applied here. ${ }^{46}$ Together, these additional analyses suggest that most of the observed charge heterogeneity is unlikely due to bacterial channel gating, and more likely to be a genuine heterogeneous charge distribution along the cell wall or a signal from natively functional ion channels.
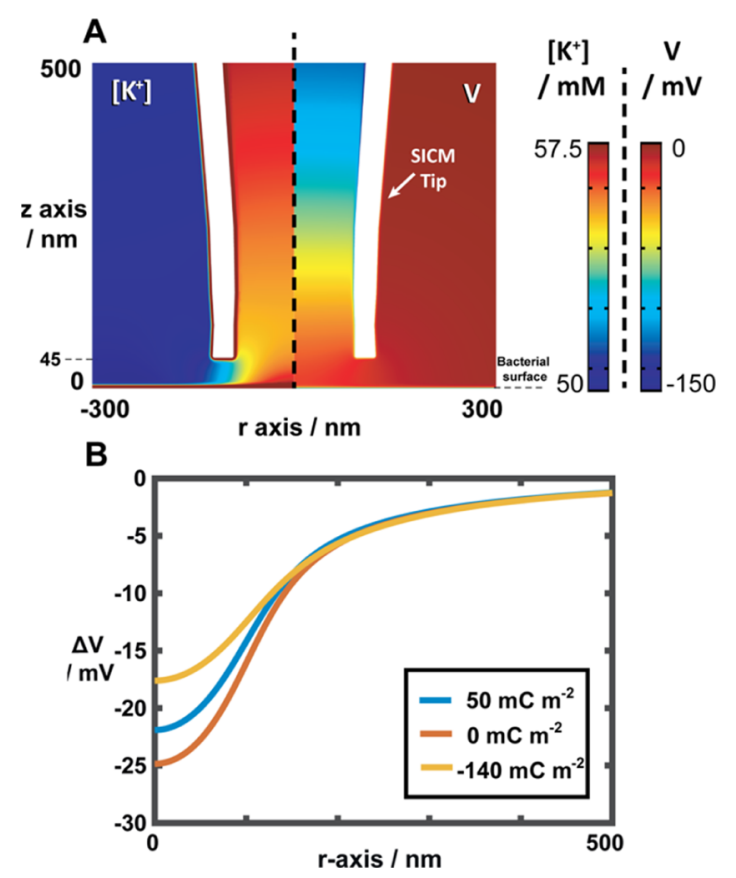

Figure 4. FEM-based simulation results of pulsed-potential SICM $\left(\mathrm{V}_{\text {Tip }}=-500 \mathrm{mV}\right.$ after $\left.20 \mathrm{~ms}\right)$ at a charged surface $\left(-140 \mathrm{mC} \mathrm{m}^{-2}\right)$. (A) Potential and $\left[\mathrm{K}^{+}\right]$distribution at surface-nanopipette interface: left panel shows the $\left[\mathrm{K}^{+}\right]$distribution and right panel shows the corresponding potential distribution vs bulk QRCE potential. The inner nanopipette radius is $95 \mathrm{~nm}$, and the nanopipette-substrate separation is $45 \mathrm{~nm}$. (B) Solution potential with respect to bulk $(\Delta V)$, from the nanopipette center radially outward at the surface-solution interface (three surface charge densities as defined), at a nanopipettesurface separation of $45 \mathrm{~nm}$. Values for different surface charge densities are shown (see the legend).

Probing the Gram-Negative Cell Envelope with Scanned-Potential SICM. Scanned-potential experiments were implemented to further confirm the findings of the pulsed-potential SICM measurements across a wider range of potentials and to further investigate the possible contribution of ion flux from voltage stimulation of cell surface efflux pumps and channels. ${ }^{47}$ In these experiments, the potential at the nanopipette was scanned in a triangular waveform across a range of values and the resulting current recorded, generating $i-V$ curves at every position of the physical scan (Figure 2B). While it increases the overall scanning time (ca. $15 \mathrm{~s} /$ position), the use of a scanned-potential protocol provides a greater depth of information (potential-resolved images presented as Movie S1 in the Supporting Information, vide infra). ${ }^{19}$ To achieve better stability of bacteria over these longer scanning periods, these experiments were performed using Cell-Tak, which provides a stronger hold on the bacteria.

Figure 5 provides a summary of typical results from the scanned-potential measurements of E. coli. Figure 5A shows $i-$ $V$ curves at selected positions over two bacteria; in all cases there is slight current rectification, with larger current magnitude at the extreme negative potential compared to positive potential $( \pm 500 \mathrm{mV})$, demonstrating that the Gramnegative bacteria surface responds to potential-scan SICM in a way similar to previous descriptions of surface-induced rectification (SIR). ${ }^{13,17,19}$ The inset in Figure 5A shows negligible current rectification between -50 and $50 \mathrm{mV}$, demonstrating that the ionic current at the nanopipette is not affected by the surface charge at the approach potential used 

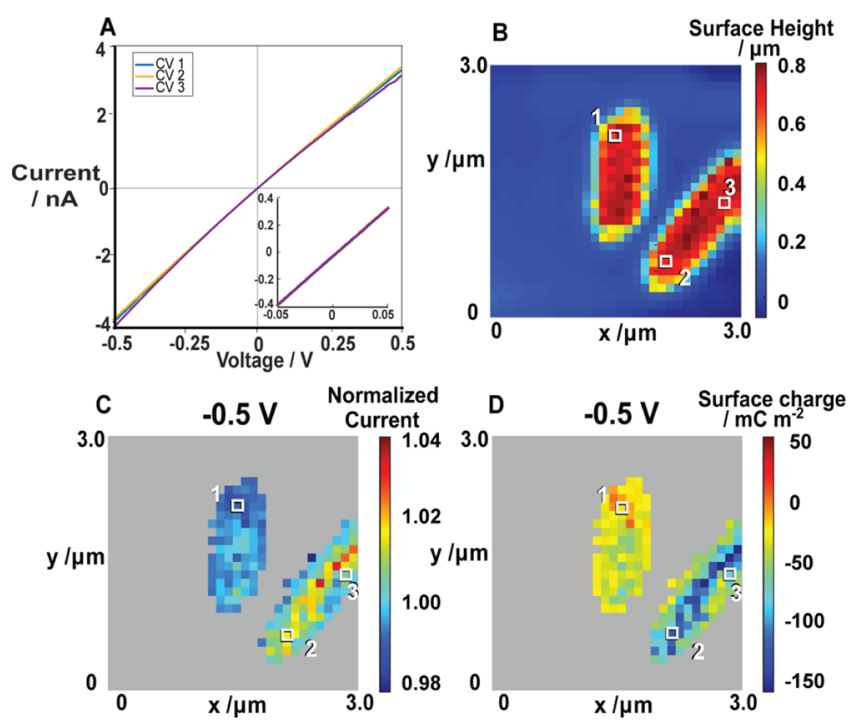

Figure 5. Scanned-potential SICM as applied to E. coli adhered with Cell-Tak in $50 \mathrm{mM}$ tris buffered $\mathrm{KCl}$ solution at $\mathrm{pH}$ 7. (A) $i-V$ curves at selected labeled positions (1-3), with the inset showing a close up of currents between -50 and $50 \mathrm{mV}$. (B) Topography maps of E. coli. (C) Normalized current maps when $V_{\text {Tip }}=-500 \mathrm{mV}$ during the potential scan. (D) Corresponding FEM-based charge density values $\left(\mathrm{mC} \mathrm{m}^{-2}\right)$ at $-500 \mathrm{mV}$. Nanopipette inner radius of $90 \mathrm{~nm}$, with 100 $\mathrm{nm}$ hopping steps (pixel separation). Scan time of $115 \mathrm{~min}$.

$(50 \mathrm{mV})$ which allows for consistent nanopipette approach distances throughout the image and the recording of the topographical map in Figure $5 \mathrm{~B}^{15}$

The normalized current and charge values at the negative extreme $(-500 \mathrm{mV})$ are shown in Figure $5 \mathrm{C}, \mathrm{D}$, respectively. Normalized current and corresponding surface charge images at the positive potential extreme $(500 \mathrm{mV})$ are given in Section SI-9, and a movie of the scan showing the normalized currents throughout the potential range (separated, potential-resolved images) is presented as Movie S1. The data in Movie S1 confirm the validity of the approach adopted: there is little contrast in the normalized current value between the bacteria and the support when the applied potential magnitude is small (insensitive to surface charge) and the contrast develops at extreme potentials (sensitive to surface charge). This is particularly striking as the E. coli bacteria were adhered using Cell-Tak (compared to PLL for Figure 3), which was found to have high positive charge values. As a consequence, to facilitate the visualization of the charge distribution over the bacteria cells, normalized currents and charge associated to the substrate (considered to be any normalized current below 0.98 , or charge above $+50 \mathrm{mC} \mathrm{m}^{-2}$ ) are removed from Figure $5 C, D$. Raw data demonstrating the full range of normalized current and charge are shown in Section SI-9, where further discussion on Cell-Tak charge properties can be found.

Figure $5 \mathrm{D}$ shows the $E$. coli bacteria have a surface charge range between -70 and $-120 \mathrm{mC} \mathrm{m}^{-2}$, in the same range of values as the pulsed SICM measurements of E. coli bacteria shown in Figure 3C. Again, the largest charge density tends to be across the central apex of the cell, and the overall charge density is visually different on the two bacteria, suggesting possible heterogeneities at the individual cell level across a cell population, which requires further analyses in future studies. Overall, there is an agreement between the results of the two SICM regimes (pulsed and scanned potentials).
As with the pulsed-potential, the scanned potential measurements also indicate local current (charge) heterogeneities on the bacteria surface (Figure 5C,D, surrounding pixel 1 and across from pixel 3). Thus, we again briefly consider whether the activation of a single MSC by the SICM measurement could occur at the largest applied nanopipette potentials (Figure 4B). MSCs, when activated, can generate currents between 40 and $75 \mathrm{pA}^{48,49}$ and multiple channels could be located within a scanned area. While the observed high current patches could possibly be natively active MSCs or ion channels, we might expect these to manifest as a discerned current increase in the measured $i-V$ profiles at a potential sufficient to drive the opening of those channels. This is neither observed in Figure 5A nor in the potential-resolved images (Movie S1, Supporting Information). Thus, there is no detectable SICM potential-induced activation of channels at larger applied potentials, and the high current patches are attributed to the heterogeneous charge over the cell surface. An interesting application of SICM in the future would be to explore whether conditions could be generated to deliberately activate ion channel function, without physical patch (contact) on the nanopipette.

Probing the Gram-Positive Cell Envelope with Pulsed-Potential SICM. We now consider experiments performed on the Gram-positive B. subtilis (using agarose for adhesion). Typical pulsed-potential SICM results are displayed in Figure 6, with SICM topographical mapping (Figure 6A)

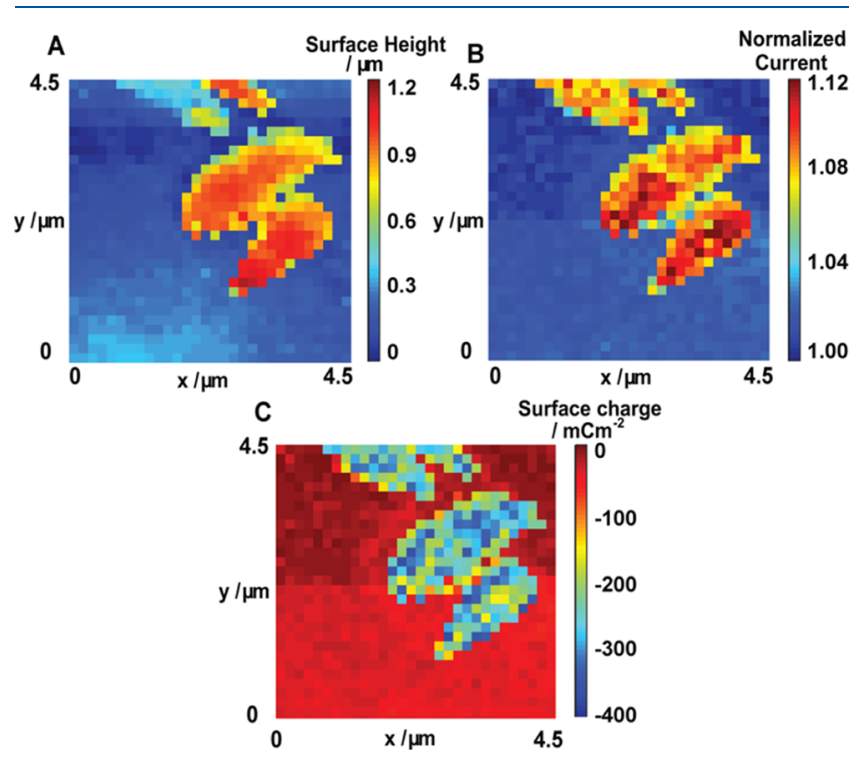

Figure 6. SICM topography (A) and normalized current (B) of $B$. subtilis $(\Delta h a g)$ on an agarose substrate, collected concurrently, along with the resulting charge density values $\left(\mathrm{mC} \mathrm{m}^{-2}\right)$ from the FEM model (C). SICM experiment conditions: $50 \mathrm{mM}$ tris buffered $\mathrm{KCl}$ at $\mathrm{pH}$ 7. $V_{\mathrm{p}}=-500 \mathrm{mV}$ and $V_{\mathrm{a}}=50 \mathrm{mV}$ utilizing a $2 \%$ feedback current threshold (see the Experimental Methods section). Nanopipette inner radius of $98 \mathrm{~nm}$, with $150 \mathrm{~nm}$ hopping steps. Scan time of $65 \mathrm{~min}$. Note that panels show raw data without any interpolation.

giving cell dimensions correspondingly well with EM images (Section SI-7). The normalized currents over the bacteria from the potential pulse (Figure 6B) are significantly higher at 1.08-1.12 compared to the E. coli case (0.98-1.05, Figure 3B), for the same experimental conditions, suggesting a high density of stationary negative charge at the interface. Intercellular heterogeneity in both cell size and normalized current 
can be observed in Figure 6, highlighting again the value of single cell measurements.

Using the model outlined in the Supporting Information (Section SI-6), surface charge density values were calculated as being between -250 and $-350 \mathrm{mC} \mathrm{m}^{-2}$, considerably greater than that for E. coli (vide supra), and similar to values in the literature from other techniques (e.g. electrophoretic mobility) for Gram-positive bacteria, where, in low concentration electrolytes, values ranging between -200 and $-500 \mathrm{mC}$ $\mathrm{m}^{-2}$ have been reported. ${ }^{50,51}$ It is important to note that while the electrolyte composition of $50 \mathrm{mM} \mathrm{KCl}$ was tolerated by our bacterial strains (see Section SI-4), it is possible that the absence of carbon sources and physiological media conditions could cause a certain degree of cellular stress, possibly affecting the charge in the bacterial cell envelope. ${ }^{52}$ However, similar high normalized currents were also found when we used a physiological media (M9m) as the electrolyte to scan B. subtilis $\Delta$ hag (Section SI-10, Figure S12).

Probing the Gram-Positive Cell Envelope with Scanned-Potential SICM. Figure 7 shows a summary of results from scanned-potential mapping of B. subtilis cells in 50 $\mathrm{mM} \mathrm{KCl}$ solution; Movie S2 (Supporting Information) shows the full potential scan. The $i-V$ curves (Figure $7 \mathrm{~A}$ ) obtained from points above the bacterium or the substrate demonstrate consistent smooth rectification profiles, attributable to SIR, ${ }^{14,19}$ but a similar behavior in a narrow potential range around $0 \mathrm{~V}$ again confirms the validity of the charge mapping strategy: in this potential region, the SICM response is insensitive to surface charge and provides a faithful map of topography.

The topography map (Figure 7B) shows two bacteria, one in whole (denoted with pixel 2), and one partially (denoted with pixel 1), which appears to be partially submerged in the agarose substrate. Although only part of the latter cell is visible in the topography map, the charge map shows more of the cell
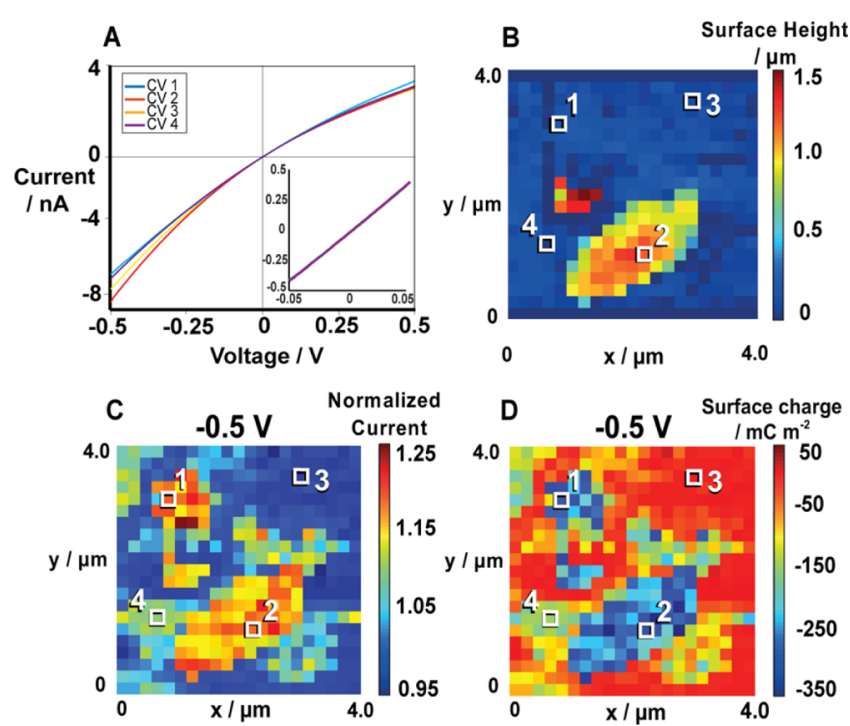

Figure 7. Scanned-potential SICM of B. subtilis ( $\Delta$ hag) in $50 \mathrm{mM}$ tris buffered $\mathrm{KCl}$ solution at $\mathrm{pH} 7$, adhered to agarose. (A) $i-V$ curves at selected labeled pixels $(1-4)$, with the inset zoomed to between -50 and $50 \mathrm{mV}$. (B) Topography maps of B. subtilis cells. (C) Normalized current maps for $V_{\text {Tip }}=-500 \mathrm{mV}$. (D) Corresponding FEM-based surface charge density values $\left(\mathrm{mC} \mathrm{m}^{-2}\right)$ at $-500 \mathrm{mV}$. Nanopipette inner radius of $93 \mathrm{~nm}$, with $200 \mathrm{~nm}$ hopping steps. Scan time of 90 min. Note that panels $(B-D)$ show raw data without any interpolation.
(Figure 7C), and a simultaneously recorded optical image (close to the resolution limit of the optical microscope used) showed the presence of two bacteria (Figure S13, Section SI11). At $V_{\text {Tip }}=-500 \mathrm{mV}$, the large normalized current ratio (Figure $7 \mathrm{C}$ ) and the corresponding negative charge density (Figure 7D) over the bacteria are seen. Significantly, however, there is a further region of negative charge density around the bacteria (Figure 7C,D, region labeled 4). We attribute these negative charged regions to the complex ion-permeable matrix known to be secreted by Gram-positive bacteria, broadly termed the extracellular polymeric substance (EPS). ${ }^{53,54} \mathrm{We}$ confirmed this through EM imaging of the bacteria, where a coating was observed over and around the cells (Section SI-7). EPS consists of polysaccharides, nucleic acids, lipids, and polypeptides $^{55}$ and can extend up to a micron from the cell surface, ${ }^{56}$ as found in this study. Our results here indicate that this material, which is also shown to aid bacterial adhesion, ${ }^{54}$ formation of biofilms, ${ }^{57}$ and nutrient trapping, ${ }^{58}$ is highly negatively charged, and that SICM charge mapping is a powerful means of assessing the regions over which EPS extends.

The difference in the ion current response between the Gram-negative E. coli and the Gram-positive B. subtilis was found to be significant (Table S9, Section SI-12) and could possibly be attributed to differences in the structure of the cell (Figure 1). For B. subtilis, the permeable and ion dense peptidoglycan layer is not shielded by an insulating outer membrane and, as such, presents a more accessible ion-rich region for the SICM tip response.

Extended FEM Model of the Gram-Positive Cell Envelope. Although the charge values recovered from the experimental data using the simplified FEM model are in good agreement with previous reports for both bacterial strains, the apparently very high charge densities for $B$. subtilis require an extended FEM model, based on a more realistic physical description of the cell wall and, in particular, the ion permeable and ion dense peptidoglycan layer. Here, we developed such an extended model specifically to account for the specific aspects of the Gram-positive cell envelope. The primary biophysical factors implemented in this extended model, treating the cell as a sphere are: the fixed charge concentration in the peptidoglycan layer $\left(\rho_{\mathrm{f}} / F\right)$, where $\rho_{\mathrm{f}}$ is the negative charge concentration and $F$ is Faraday's constant, the thickness of the peptidoglycan cell wall ( $t_{\text {wall }}$, see Section SI-6.2) and the mobility of counter ions within it $\left(\mu_{\text {wall }}\right)$, relative to that in solution. It was previously found that co-ions are excluded from the peptidoglycan cell wall of Bacillus brevis at electrolyte concentrations similar to those employed in our experiments, ${ }^{31}$ and hence the model assumed no $\mathrm{Cl}^{-}$partitioning to the cell wall. A full description of the extended model can be found in Sections SI-6 and SI-13.

Using this extended model, we re-simulated the SICM experiments as applied to $B$. subtilis, with results shown in Figure 8. Figure $8 \mathrm{~A}$ shows the concentration profile of $\mathrm{K}^{+}$ions in the vicinity of the nanopipette end and cell wall. At the approach potential $\left(V_{\mathrm{a}}=50 \mathrm{mV}\right),\left[\mathrm{K}^{+}\right]$is more or less uniform, except in the double layer of the charged interfaces. With a negative pulse potential $\left(V_{\mathrm{p}}=-500 \mathrm{mV}\right), \mathrm{K}^{+}$is drawn from the cell wall into the nanopipette, leading to an increase in $\left[\mathrm{K}^{+}\right]$in the vicinity of the nanopipette end. The corresponding potential distributions for these cases are shown in Figure 8B. This is shown in more detail in Figure 8C, where the potential distribution over the thickness of the cell wall and just into the 

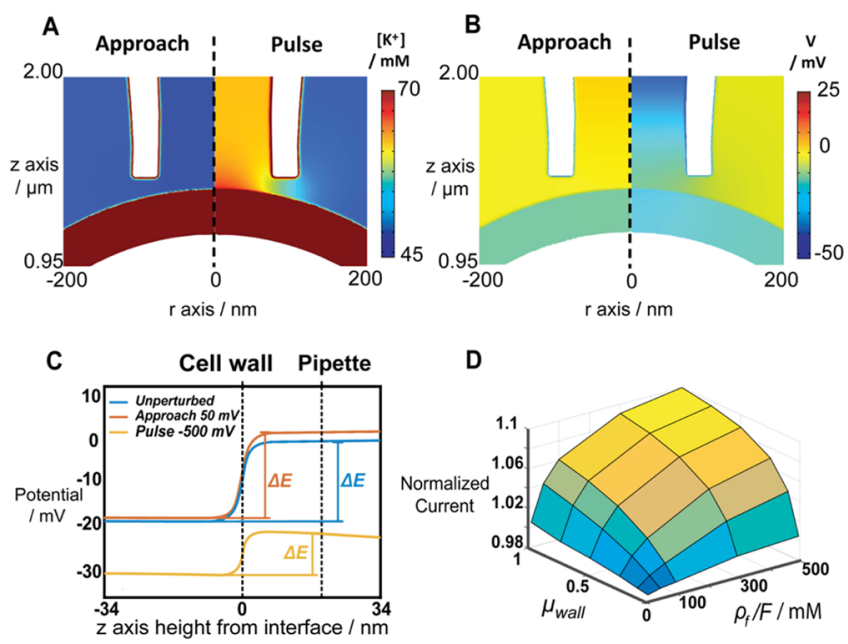

D
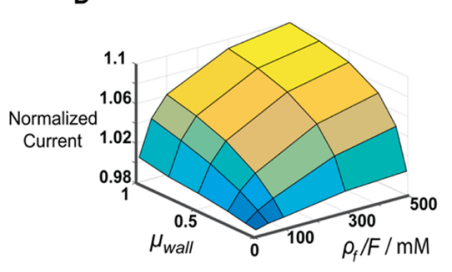

Figure 8. FEM simulations of pulsed-potential SICM at the Grampositive cell surface with an assumed stationary charge concentration $\left(\rho_{\mathrm{f}} / F\right)$ of $100 \mathrm{mM}$ in the peptidoglycan layer. The other parameters were: peptidoglycan cell wall (substrate) thickness of $70 \mathrm{~nm}$; nanopipette-substrate distance of $20 \mathrm{~nm}$; dielectric relative permittivity of the cell wall of 20; and relative ionic mobility within the cell wall compared to solution $\left(\mu_{\text {wall }}\right)$ of $1 .(\mathrm{A})\left[\mathrm{K}^{+}\right]$and (B) potential distribution at the approach potential $(50 \mathrm{mV}$, left $)$ and the pulse potential $(-500 \mathrm{mV}$, right). (C) Potential-distance profiles across the cell wall-solution interface (at the cylindrical symmetry axis): with no perturbation (blue); at the approach potential (orange, $V_{\text {Tip }}=50 \mathrm{mV}$ ); and at the pulse potential (yellow, $V_{\text {Tip }}=-500 \mathrm{mV}$ ). For these plots, the cell wall interface is at $0 \mathrm{~nm}$, with negative values of $z$ into the cell wall and positive values into the solution (and positions of the cell wall and nanopipette marked by the vertical dashed lines). (D) Normalized SICM current (at nanopipette potential of $-500 \mathrm{mV}$ ) as a function of $\mu_{\text {wall }}$ and $\rho_{\mathrm{f}} / F$.

solution ( $z$-coordinate) is shown for the cases of no applied potential and $V_{\text {Tip }}$ set at 50 or $-500 \mathrm{mV}$. In all these cases, the interior of the cell wall $(-34$ to $0 \mathrm{~nm})$ reaches the Donnan equilibrium, noted by the plateau in the potential value. The potential difference between the cell wall and nanopipette end $(\Delta E$ in Figure $8 \mathrm{C}$ ) are $-17.5 \mathrm{mV}$ for the unperturbed case $\left(V_{\text {Tip }}=0 \mathrm{mV}\right),-19 \mathrm{mV}$ when the approach potential is applied $\left(V_{\text {Tip }}=50 \mathrm{mV}\right)$ and $-9.4 \mathrm{mV}$ under the pulsed-potential $\left(V_{\text {Tip }}\right.$ $=-500 \mathrm{mV})$. A summary plot, indicating how the normalized nanopipette current varies with wall charge density $\left(\rho_{\mathrm{f}} / F\right)$ and ionic mobility within the cell wall $\left(\mu_{\text {wall }}\right)$, with all other parameters fixed to literature estimated values (Section SI-6), is shown in Figure 8D.

These simulation results show that the normalized SICM current values are dependent on $\rho_{\mathrm{f}} / F$ and $\mu_{\text {wall }}$, as expected. With increasing charge density and/or the mobility of ions in the peptidoglycan cell wall, the normalized current ratio increases, reaching a maximum value of $c a$. 1.1, close to that observed experimentally for the B. subtilis cells over most of the cell surface (Figures 6 and 7). Modifying the cell wall thickness within the range observed by EM (Figure S14B) causes only minor changes in the simulated normalized currents because the perturbation caused by the SICM probe only extends into the outermost region of the cell wall (Figure 8B), as also seen with permeable abiotic substrates. ${ }^{34}$

An interesting avenue for future exploration would be to analyze in more detail the current-time transients in pulsedpotential measurements and apply double pulses, to transiently drive the cell wall (substrate) interface out of equilibrium with
SICM, and to monitor its return to equilibrium. Analysis of similar scanning electrochemical microscopy studies has enabled the relative diffusion coefficient and partition coefficient to be determined independently in a single measurement, without the tip contacting the sample. ${ }^{59,60}$ To ensure sensitivity to the cell wall, it may also be beneficial to explore the use of differential concentration SICM, with a different concentration of the electrolyte in the nanopipette compare to that in the bulk solution. ${ }^{39}$

\section{CONCLUSIONS}

Here, we demonstrated the application of SICM to the study of the ionic environment of live Gram-positive and Gramnegative bacteria. Several methods used for adhering bacteria to the glass substrate allow SICM while maintaining cell viability. The adherents selected were not shown to affect the bacteria ionic environment, and similar SICM current responses were seen for the same strains across different substrates. Using FEM simulations to model the influence of the SICM nanopipette tip on the cell wall interface, we were able to understand and account for the potential and hydrodynamic pressure perturbations applied to the bacteria, showing that the SICM tip, at the conditions employed here, can be considered as non-inductive to cell physiology, allowing surface charge of both strains to be analyzed using SICM and FEM.

In-line with previous bulk measurements, we found that both Gram-positive and Gram-negative bacteria display negative surface charges, with Gram-positive B. subtilis having a significantly more negative charge than the Gram-negative $E$. coli. This was confirmed by using two SICM regimes, a pulsedpotential and a scanned-potential regime, both of which yield similar charge results. The scanned-potential regime also produced similar charge values at both potential extremes, demonstrating that the direction of polarity did not considerably influence the calculated surface charge. The application of FEM simulations allowed us to calculate surface charge for both bacterial types, and an extended model allowed first insights into capturing the impact of peptidoglycan layers on SICM measurements performed on Gram-positive bacteria. This extended model has potential to be developed further to capture complex transport phenomena at the Gram-positive cell wall.

By utilizing small nanopipettes as the SICM tip, we were able to visualize charge heterogeneity between and across the individual bacteria and the charge distribution over the secreted EPS layer. This high spatial resolution, coupled with an extended model of the Gram-negative bacteria cell wall, allowed initial insights to the intricate interplay between the biophysical and SICM parameters. We also suggest that SICM has the potential to be applied in a more intrusive manner to investigate ion channels within the cell wall without destroying the integrity of the cell structure, a technique that we envisage could be similar to a form of non-contact patch clamping. Thus, the presented methodology will pave the way to a more thorough understanding of the interconnection between cellular physiology and bioelectrical microenvironment of cells, benefiting a broad range of research areas including cell biology, bacterial adhesion, antibiotic resistance, and biofilm formation, and making a significant impact on life science research and development. 


\section{ASSOCIATED CONTENT}

\section{(1) Supporting Information}

The Supporting Information is available free of charge at https://pubs.acs.org/doi/10.1021/acs.analchem.0c03653.

Description of the bacterial strains, details of bacteria culture and SICM sample preparation, bacteria cell viability study, nanopipette fabrication and characterization, details of numerical simulations, EM of bacteria cells, further analyses of the scanned- and pulse-potential E. coli scans, and statistical analyses of normalized currents recorded over the two different bacterial strains (PDF)

Potential-resolved video constructed from scannedpotential SICM scan of E. coli (MOV)

Potential-resolved video constructed from scannedpotential SICM scan of B. subtilis (MOV)

\section{AUTHOR INFORMATION}

\section{Corresponding Authors}

Orkun S. Soyer - Bio-Electrical Engineering Innovation Hub and School of Life Sciences, University of Warwick, Coventry CV4 7AL, U.K.; Email: o.soyer@warwick.ac.uk

Patrick R. Unwin - Bio-Electrical Engineering Innovation $\mathrm{Hub}$ and Department of Chemistry, University of Warwick, Coventry CV4 7AL, U.K.; (1) orcid.org/0000-0003-31062178; Email: p.r.unwin@warwick.ac.uk

\section{Authors}

Kelsey Cremin - Bio-Electrical Engineering Innovation Hub, Department of Chemistry, Molecular Analytical Science Centre for Doctoral Training (MAS CDT), and School of Life Sciences, University of Warwick, Coventry CV4 7AL, U.K.

Bryn A. Jones - Department of Chemistry, University of Warwick, Coventry CV4 7AL, U.K.

James Teahan - Department of Chemistry and Molecular Analytical Science Centre for Doctoral Training (MAS CDT), University of Warwick, Coventry CV4 7AL, U.K.

Gabriel N. Meloni - Bio-Electrical Engineering Innovation $\mathrm{Hub}$ and Department of Chemistry, University of Warwick, Coventry CV4 7AL, U.K.; (1) orcid.org/0000-0002-29502464

David Perry - Department of Chemistry, University of Warwick, Coventry CV4 7AL, U.K.; (1) orcid.org/00000002-8630-4798

Christian Zerfass - Bio-Electrical Engineering Innovation $\mathrm{Hub}$ and School of Life Sciences, University of Warwick, Coventry CV4 7AL, U.K.

Munehiro Asally - Bio-Electrical Engineering Innovation Hub and School of Life Sciences, University of Warwick, Coventry CV4 7AL, U.K.; (1) orcid.org/0000-0002-8273-7617

Complete contact information is available at: https://pubs.acs.org/10.1021/acs.analchem.0c03653

\section{Author Contributions}

K.C., B.A.J., J.T., and G.N.M. contributed equally. Methods were designed by K.C., B.A.J., and G.N.M., with experiments performed by K.C. and B.A.J. Simulations were developed by J.T., G.N.M., and D.P. and performed by K.C. and J.T. C.Z. and M.A. provided support and advice for biological materials, protocols, and analysis. P.R.U. and O.S.S. conceived the study, supervised, and helped with data interpretations. The manuscript was written by K.C., G.N.M., J.T., O.S.S., and P.R.U.

Notes

The authors declare no competing financial interest.

\section{ACKNOWLEDGMENTS}

J.T. and K.C. thank the EPSRC for support through MAS CDT, grant number EP/L015307/1. B.A.J. thanks EPSRC/ Unilever for an iCASE award. G.N.M. acknowledges support from the European Union's Horizon 2020 research and innovation program under the Marie Skłodowska-Curie grant agreement 790615 (FUNNANO). P.R.U. thanks the Royal Society for support through a Wolfson Research Merit Award. The authors would all like to acknowledge the support of the Bio-Electrical Engineering Innovation Hub, University of Warwick, funded by the UK's Biological and Biotechnological Sciences (grant no. BB/S506783/1) and Engineering and Physical Sciences Research Councils. The authors acknowledge the Kearns laboratory, from Indiana University, who developed the B. subtilis mutants used that we obtained through M.A. We also gratefully acknowledge Corinne Bailey and Saskia Bakker, from the University of Warwick Advanced Bioimaging platform, for assistance with the EM imaging of the bacterial strains, and Ian McPherson for fruitful discussions. Finally, we wish to acknowledge Teuta Pillizota, from the University of Edinburgh for fruitful discussions and support regarding adhesion of bacteria to substrates.

\section{REFERENCES}

(1) Hansma, P.; Drake, B.; Marti, O.; Gould, S.; Prater, C. Science 1989, 243, 641-643.

(2) Korchev, Y. E.; Bashford, C. L.; Milovanovic, M.; Vodyanoy, I.; Lab, M. J. Biophys. J. 1997, 73, 653-658.

(3) Korchev, Y. E.; Negulyaev, Y. A.; Edwards, C. R. W.; Vodyanoy, I.; Lab, M. J. Nat. Cell Biol. 2000, 2, 616-619.

(4) Chen, C.-C.; Zhou, Y.; Morris, C. A.; Hou, J.; Baker, L. A. Anal. Chem. 2013, 85, 3621-3628.

(5) Zhou, L.; Gong, Y.; Hou, J.; Baker, L. A. Anal. Chem. 2017, 89, 13603-13609.

(6) Zhou, L.; Gong, Y.; Sunq, A.; Hou, J.; Baker, L. A. Anal. Chem. 2016, 88, 9630-9637.

(7) Huang, K.; Zhou, L.; Alanis, K.; Hou, J.; Baker, L. A. Chem. Sci. 2020, 11, 1307-1315.

(8) Ida, H.; Takahashi, Y.; Kumatani, A.; Shiku, H.; Matsue, T. Anal. Chem. 2017, 89, 6015-6020.

(9) Simeonov, S.; Schäffer, T. E. Nanoscale 2019, 11, 8579-8587.

(10) Takahashi, Y.; Zhou, Y.; Miyamoto, T.; Higashi, H.; Nakamichi, N.; Takeda, Y.; Kato, Y.; Korchev, Y.; Fukuma, T. Anal. Chem. 2020, 92, 2159-2167.

(11) Shevchuk, A. I.; Gorelik, J.; Harding, S. E.; Lab, M. J.; Klenerman, D.; Korchev, Y. E. Biophys. J. 2001, 81, 1759-1764.

(12) Momotenko, D.; McKelvey, K.; Kang, M.; Meloni, G. N.; Unwin, P. R. Anal. Chem. 2016, 88, 2838-2846.

(13) Sa, N.; Baker, L. A. J. Am. Chem. Soc. 2011, 133, 10398-10401.

(14) Sa, N.; Lan, W.-J.; Shi, W.; Baker, L. A. ACS Nano 2013, 7, 11272-11282.

(15) McKelvey, K.; Kinnear, S. L.; Perry, D.; Momotenko, D.; Unwin, P. R. J. Am. Chem. Soc. 2014, 136, 13735-13744.

(16) Page, A.; Perry, D.; Young, P.; Mitchell, D.; Frenguelli, B. G.; Unwin, P. R. Anal. Chem. 2016, 88, 10854-10859.

(17) Zhu, C.; Zhou, L.; Choi, M.; Baker, L. A. ChemElectroChem 2018, 5, 2986-2990.

(18) Perry, D.; Al Botros, R.; Momotenko, D.; Kinnear, S. L.; Unwin, P. R. ACS Nano 2015, 9, 7266-7276. 
(19) Perry, D.; Paulose Nadappuram, B.; Momotenko, D.; Voyias, P. D.; Page, A.; Tripathi, G.; Frenguelli, B. G.; Unwin, P. R. J. Am. Chem. Soc. 2016, 138, 3152-3160.

(20) Maddar, F. M.; Perry, D.; Brooks, R.; Page, A.; Unwin, P. R. Anal. Chem. 2019, 91, 4632-4639.

(21) Kang, M.; Perry, D.; Bentley, C. L.; West, G.; Page, A.; Unwin, P. R. ACS Nano 2017, 11, 9525-9535.

(22) Schofield, Z.; Meloni, G. N.; Tran, P.; Zerfass, C.; Sena, G.; Hayashi, Y.; Grant, M.; Contera, S. A.; Minteer, S. D.; Kim, M.; Prindle, A.; Rocha, P.; Djamgoz, M. B. A.; Pilizota, T.; Unwin, P. R.; Asally, M.; Soyer, O. S. J. R. Soc. Interface 2020, 17, 20200013.

(23) Liu, H.; Fang, H. H. P. Biotechnol. Bioeng. 2002, 80, 806-811.

(24) Tedja, R.; Lim, M.; Amal, R.; Marquis, C. ACS Nano 2012, 6, 4083-4093.

(25) Bahar, A. A.; Liu, Z.; Garafalo, M.; Kallenbach, N.; Ren, D. Pharmaceuticals 2015, 8, 696-710.

(26) Davis, K. M.; Isberg, R. R. BioEssays 2016, 38, 782-790.

(27) Coico, R. Current Protocols in Microbiology; John Wiley \& Sons, Inc.: Hoboken, NJ, USA, NJ, USA, 2005.

(28) Voet, D.; Voet, J. G. Biochemistry; John Wiley \& Sons, 2011.

(29) Corpe, W. A. In Adhesion in Biological Systems; Manly, R. S., Ed.; Academic Press, Inc.: New York, 1970.

(30) Dickson, J. S.; Koohmaraie, M.; Hruska, R. L. Appl. Environ. Microbiol. 1989, 55, 832.

(31) van der Wal, A.; Norde, W.; Zehnder, A. J. B.; Lyklema, J. Colloids Surf., B 1997, 9, 81-100.

(32) Ahimou, F.; Denis, F. A.; Touhami, A.; Dufrêne, Y. F. Langmuir 2002, 18, 9937-9941.

(33) Marlière, C.; Dhahri, S. Nanoscale 2015, 7, 8843-8857.

(34) Payne, N. A.; Dawkins, J. I. G.; Schougaard, S. B.; Mauzeroll, J. Anal. Chem. 2019, 91, 15718-15725.

(35) Seifert, J.; Rheinlaender, J.; Novak, P.; Korchev, Y. E.; Schäffer, T. E. Langmuir 2015, 31, 6807-6813.

(36) Rheinlaender, J.; Geisse, N. a.; Proksch, R.; Schäffer, T. E. Langmuir 2011, 27, 697-704.

(37) Mukherjee, S.; Babitzke, P.; Kearns, D. B. J. Bacteriol. 2013, 195, 297-306.

(38) Benn, G.; Pyne, A. L. B.; Ryadnov, M. G.; Hoogenboom, B. W. Analyst 2019, 144, 6944-6952.

(39) Perry, D.; Page, A.; Chen, B.; Frenguelli, B. G.; Unwin, P. R. Anal. Chem. 2017, 89, 12458-12465.

(40) Momotenko, D.; Girault, H. H. J. Am. Chem. Soc. 2011, 133, 14496-14499.

(41) Rao, D. G. Introduction to Biochemical Engineering; Tata McGraw-Hill, 2005.

(42) Zhu, Z.; Ye, Z.; Zhang, Q.; Zhang, J.; Cao, F. Electrochem. Commun. 2018, 88, 47-51.

(43) Nadappuram, B. P.; McKelvey, K.; Al Botros, R.; Colburn, A. W.; Unwin, P. R. Anal. Chem. 2013, 85, 8070-8074.

(44) Dufrêne, Y. F. mBio 2014, 5, 1-14.

(45) Stratford, J. P.; Edwards, C. L. A.; Ghanshyam, M. J.; Malyshev, D.; Delise, M. A.; Hayashi, Y.; Asally, M. Proc. Natl. Acad. Sci. U.S.A. 2019, 116, 9552-9557.

(46) Martinac, B.; Buechner, M.; Delcour, A. H.; Adler, J.; Kung, C. Cell Biol. 1987, 84, 2297-2301.

(47) Babu, M.; Bundalovic-Torma, C.; Calmettes, C.; Phanse, S.; Zhang, Q.; Jiang, Y.; Minic, Z.; Kim, S.; Mehla, J.; Gagarinova, A.; Rodionova, I.; Kumar, A.; Guo, H.; Kagan, O.; Pogoutse, O.; Aoki, H.; Deineko, V.; Caufield, J. H.; Holtzapple, E.; Zhang, Z.; Vastermark, A.; Pandya, Y.; Lai, C. C.-1.; El Bakkouri, M.; Hooda, Y.; Shah, M.; Burnside, D.; Hooshyar, M.; Vlasblom, J.; Rajagopala, S. V.; Golshani, A.; Wuchty, S.; F Greenblatt, J.; Saier, M.; Uetz, P.; F Moraes, T.; Parkinson, J.; Emili, A. Nat. Biotechnol. 2018, 36, 103112.

(48) Andersson, M.; Okeyo, G.; Wilson, D.; Keizer, H.; Moe, P.; Blount, P.; Fine, D.; Dodabalapur, A.; Duran, R. S. Biosens. Bioelectron. 2008, 23, 919-923.

(49) Martinac, B.; Saimi, Y.; Kung, C. Physiol. Rev. 2008, 88, 14491490.
(50) Poortinga, A. T.; Bos, R.; Norde, W.; Busscher, H. J. Surf. Sci. Rep. 2002, 47, 1-32.

(51) Bulard, E.; Fontaine-Aupart, M.-P.; Dubost, H.; Zheng, W.; Bellon-Fontaine, M.-N.; Herry, J.-M.; Bourguignon, B. Langmuir 2012, 28, 17001-17010.

(52) Flores-Kim, J.; Darwin, A. J.; Flores-Kim, J. Virulence 2015, 5, $835-851$.

(53) Xiao, Y.; Zhang, E.; Zhang, J.; Dai, Y.; Yang, Z.; Christensen, H. E. M.; Ulstrup, J.; Zhao, F. Sci. Adv. 2017, 3, No. e1700623.

(54) Tsuneda, S.; Aikawa, H.; Hayashi, H.; Yuasa, A.; Hirata, A. FEMS Microbiol. Lett. 2003, 223, 287-292.

(55) Flemming, H.-C. Microorganisms 2016, 4, 41.

(56) Avinash, M.; Bhavanth, J. In The Prokaryotes: Applied Bacteriology and Biotechnology; Rosenberg, E., DeLong, E. F., Thompson, F., Lory, S., Stackebrandt, E., Eds.; Springer Berlin Heidelberg: Berlin, 2013.

(57) Jachlewski, S.; Jachlewski, W. D.; Linne, U.; Bräsen, C.; Wingender, J.; Siebers, B. Front. Bioeng. Biotechnol. 2015, 3, 1-11.

(58) Harimawan, A.; Ting, Y.-P. Colloids Surf., B 2016, 146, 459467.

(59) Gonsalves, M.; Barker, A. L.; Macpherson, J. V.; Unwin, P. R.; O'Hare, D.; Winlove, C. P. Biophys. J. 2000, 78, 1578-1588.

(60) Barker, A. L.; Unwin, P. R. J. Phys. Chem. B 2001, 105, 1201912031. 